\title{
La Oscurana (Cajamarca), un bosque relicto más para conservar en las vertientes occidentales andinas del norte del Perú
}

\section{La Oscurana (Cajamarca), one more relict forest to be conserved from the western Andean slopes in northern Peru}

\author{
Ana M. Juárez*, José E. Ayasta*, Roxana P. Aguirre* y Eric F. Rodríguez**
}

Publicación: 08/12/2005

\section{Resumen}

Se presenta un análisis de la composición florística, formas de vida, importancia y estrategias para la conservación de un bosque relicto de la Vertiente Occidental Andina del norte del Perú, descrito por primera vez y denominado La Oscurana (caserío El Nogal, distrito Bolívar, provincia San Miguel de Pallaques, Cajamarca; $6^{\circ} 90^{\prime} \mathrm{S}$ y $79^{\circ} 08^{\prime} \mathrm{O}, 2000-2800 \mathrm{~m}$ ). El análisis preliminar incluye 85 familias, 169 géneros y 258 especies de plantas vasculares, de las cuales 15 familias corresponden a Pteridophyta y 70 a Magnoliophyta. En los ámbitos genérico y específico las Asteraceae son las mejor representadas de las Magnoliopsida y las Orchidaceae de las Liliopsida. Las formas de vida predominantes en cuanto a familia se reúnen en seis categorías: hierbas con 29 familias (34\%), arbustos con $14(16 \%)$, árboles con $9(11 \%)$, trepadoras/lianas con $9(11 \%)$, hierbas/arbustos con 7 (8\%) y hierbas/epífitas con 5 familias (6\%). Se encontró que además de albergar una diversidad excepcional y brindar utilidad económica local, muchas de las especies no están registradas en las vertientes occidentales andinas del norte y del sur. Por la importancia que posee y por ser uno de los últimos bosques relictos existentes, la conservación legal de éste frágil ecosistema es urgente. Se propone que debe ser declarada zona de protección o intangible y que su conservación debe ser integral, involucrando a científicos, Estado, gobiernos regionales, gobiernos locales y población.

Palabras clave: bosque montañoso, bosque relicto, Cajamarca, diversidad florística, Vertiente Occidental Andina

\section{Abstract}

Floristic composition, the life forms, the importance and strategies for the conservation of a newly discovered relict forest in the north of Peru, called La Oscurana, are analyzed (El Nogal Hamlet, Bolívar District, San Miguel de Pallaques Province, Cajamarca Department; 6 90' S y 79 08' W, 2000 $2800 \mathrm{~m}$ ). The preliminary analysis includes 85 families, 169 genera and 258 species of vascular plants, 15 families are Pteridophyta and 70 Magnoliophyta. The family with the most genera and species are the Asteraceae in the Magnoliopsida, and the Orchidaceae in the Liliopsida. The predominant life forms of the families can be divided in 6 different categories: herbs 29 (34\%), shrubs $14(16 \%)$, trees $9(11 \%)$, lianas $9(11 \%)$, herbs/shrubs $7(8 \%)$ and epiphytes $5(6 \%)$. The exceptional diversity and the local economic use are discussed. The conservation of this fragile and important ecosystem is urgent. It is one of the last existing relict forests in the region. The forest should be declared as a protected zone. Scientists, state officials, members of the regional government and the local people should work out together in the conservation plan.

Keywords: Montane forests, relict forests, Cajamarca, floristic diversity, Andean Western slope.

\section{Introducción}

Actualmente la biodiversidad sigue sufriendo cambios dramáticos. Se encuentra en un proceso acelerado de destrucción, quedando desconocida o poco estudiada mucha de ella.

(*) Facultad de Biología, Universidad Nacional Pedro Ruiz Gallo, Lambayeque, Perú

(**) Herbarium Truxillense (HUT), Universidad Nacional de Trujillo, Trujillo, Perú.

E-mail: efrr@unitru.edu.pe
La causa principal es la deforestación extensiva e irreversible que la flora (v.g. los bosques) soporta principalmente en la zona tropical. Se calcula que estos bosques ocupan menos del 10\% de la superficie terrestre; $\sin$ embargo, albergan las tres cuartas parte de todas las especies del planeta, perdiéndose anualmente el 1,1\% aproximadamente (Gentry, 1994; en Vásquez, 1995). La explicación de la deforestación producida es histórica y atribui- 
ble directa y casi exclusivamente a la ganadería y a la ampliación de la frontera agrícola (agricultura migratoria) por parte de colonos (Dourejanni, 1990), con el único fin de ganar tierras para satisfacer la necesidad primaria dealimentación yen menor grado a la explotación forestal.

El Perú no es la excepción en la pérdida de biodiversidad. Particularmente afronta este hecho la parte norte de las vertientes occidentales andinas, perteneciente a la región biogeográfica denominada zona de AmotapeHuancabamba (Weigend, 2002). Muchas áreas aún no han sido estudiadas en detalle y otras consideradas como forestales que no son protegidas por el Estado, necesitan de inventarios biológicos y conservación urgente, pues el impacto humano es rápido e irreversible. Se conocen valiosos estudios de floras o flórulas aisladas de esta parte del país, que servirán en el futuro cuando se tenga la disposición de efectuar programas de conservacion (Sagástegui \& Dillon, 1991; Dillon, 1993, 1994, 1995; Sagástegui, 1994; Sagástegui et al., 1995; Sagástegui, 1995; López, 1993, 1995a, 1995b, 1998a, 1998b; Sagástegui et al., 1999; Sagástegui et al., 2003).

En este contexto, una de las provincias que menos atención botánica ha recibido es la de San Miguel de Pallaques (Cajamarca), a pesar de que presenta numerosas variaciones geomorfológicos y edáficas que han dado origen a un mosaico de hábitats, reflejándose en la cobertura vegetal y grado de endemismo (Ritter, 1981; Sagástegui \& Sánchez, 1991; Luteyn, 1992; Robinson, 1993; Sagástegui, 1994; Hensold, 1999; Rodríguez et al., 2000, 2002). Los únicos trabajos florísticos que se conocen son los de Alvítez (1977), quien hace un estudio preliminar de los aspectos fitogeográficos para esta provincia; Dillon et al. (1994) da a conocer la flora del bosque Tongod (San Miguel) caracterizado por la presencia de Podocarpus oleifolius D. Don., y Rodríguez et al. (2002) quienes dan a conocer un inventario para la provincia con 103 familias, 330 géneros y 573 especies de un total de 1500 registros.
En San Miguel de Pallaques existen bosques montanos nublados interesantes, tales como los bosques de Tongod-Quellacorco y Taulis, muy intervenidos actualmente y el bosque relicto de La Oscurana en el distrito de Bolívar; no obstante que a su alrededor existen áreas con impacto ambiental y una colonización desordenada, se encuentra casi íntegro. Este último fragmento de bosque húmedo nunca antes fue estudiado. Sólo se conocen colecciones antiguas efectuadas en los alrededores por Raimondi y Weberbauer (Weberbauer, 1945); así como colecciones modernas realizadas por A. Sagástegui, I. Sánchez, E. Alvítez, J. Mostacero, S. Leiva, E. Rodríguez, S. Llatas y M. Weigend, entre otros. La conservación legal de este frágil ecosistema es urgente y prioritaria.

El presente trabajo forma parte de la flora de esta provincia y el objetivo es dar a conocer este nuevo ecosistema con alta diversidad florística, así como plantear estrategias para su conservación legal.

\section{Material y métodos}

El estudio se realizó en el bosque relicto húmedo de La Oscurana, Caserío El Nogal, a $9 \mathrm{~km}$ del pueblo de Bolívar, distrito de Bolívar, provincia de San Miguel de Pallaques (Dpto. Cajamarca, 6 90' S y 79 08' W, 2000-2800 m; Fig. 1). Este ecosistema está ubicado sobre un acantilado rocoso-escarpado, debajo de la catarata «El Chivo» y es cruzado por la quebrada del mismo nombre proveniente de Niepos, la cual ayuda a mantener la humedad, coadyuvado por las nubes estratos que lo cubren gran parte del año y las lluvias estacionales (Fig. 2). El área estudiada presenta una inclinación de $35 \%$ de pendiente y tiene 65 ha de extensión.

El material botánico comprende ca. 550 registros basados en el estudio de colecciones efectuadas por los autores entre los años 2002 al 2004 y depositadas en los herbarios: HAO, HUT, PRG, USM. La colección se efectúo de acuerdo con las técnicas y metodología convencional (Womersley, 1981; Rodríguez \& 


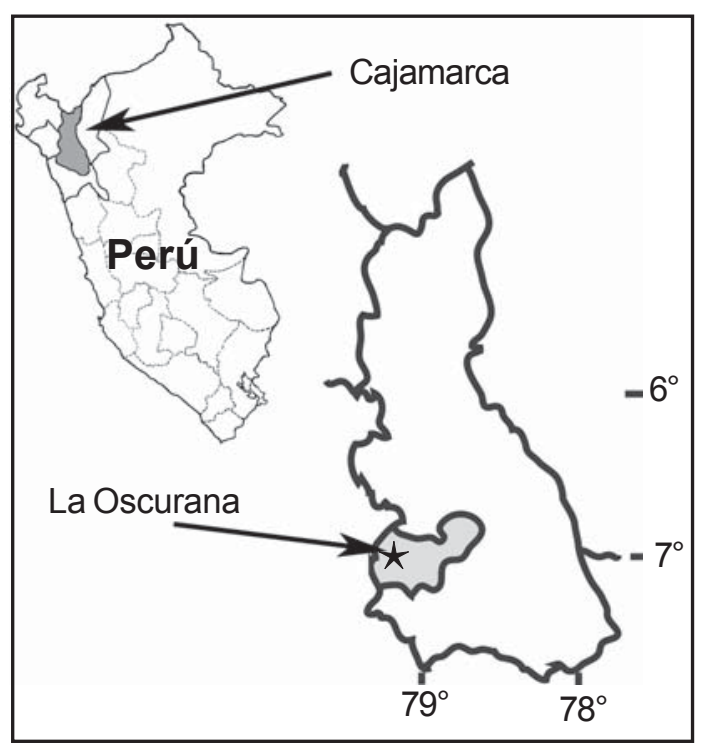

Figura 1. Ubicación geográfica del área de estudio en la provincia de San Miguel de Pallaques, Dpto. de Cajamarca, Perú.

Rojas, 2002) y los muestreos según Foster et al. (1996). La confirmación y en algunos casos la determinación taxonómica fue efectuada por especialistas y por comparación con los ejemplares existentes en el Herbario Antenor Orrego (HAO), Herbarium Truxillense (HUT), Herbario de la Universidad Pedro Ruiz Gallo (PRG) y Herbario de la Universidad de San Marcos (USM). Se consultó bibliografía disponible para cada taxón (e.g. Macbride, 1936 y siguientes; Tryon \& Stolze, 1989-1994; Brako \& Zarucchi, 1993). En el ordenamiento sistemático se siguió a Tryon \& Tryon (1982) para las Pteridophyta, y Cronquist (1981, 1988) para las Magnoliophyta. También se consigna la composición florística general, formas de vida, la riqueza máxima referida a familias, géneros y especies; así como datos etnobotánicos a través de informantes locales.

El bosque La Oscurana, representa un fragmento de la vegetación continua que existió antiguamente en esta parte del Perú. Por la composición florística, tomando en cuenta los géneros registrados, presenta similitud biogeográfica con el bosque Monteseco, ubicado en la provincia de Santa Cruz, Cajamarca (Sagástegui \& Dillon, 1991; Dillon, 1993;
Sagástegui et al., 2003). Por la proximidad geográfica, definitivamente estos dos bosques nublados han formado un pasado continuo. En estos relictos de bosque se encuentran comunidades con especies no registradas en otros bosques de las vertientes occidentales andinas del norte y del sur, solamente en Monteseco (Sagástegui et al., 2003). La actividad antrópica, con fines agropecuarios, ha sido y es la principal causante de la pérdida de biodiversidad y de la fragmentación de los bosques. El aspecto más sobresaliente de la conformación del área de estudio está relacionado con las variaciones geomorfológicas y edáficas, las cuales son contrastantes y han dado origen a una marcada diversidad de hábitats observables desde los 2000 a $2800 \mathrm{~m}$, sumado a esto la historia geológica (fragmentación de hábitat, contacto secundario) lo cual determina la diversidad vegetal. La Oscurana Pertenece a la formación de bosque muy húmedo Montano Bajo Tropical (bmh-MBT) por sus características fisiográficas orientales, a pesar de estar enclavado en la vertiente occidental.

\section{Resultados}

\section{Composición florística y formas de vida}

El análisis incluye 85 familias, 169 géneros y 258 especies de plantas vasculares, de las cuales 15 familias corresponden a Pteridophyta y 70 a Magnoliophyta (Tabla 1). No se han registrado Gimnospermae y las Magnoliopsida se constituyen en las más diversas. En la tabla 2, las familias con mayor diversidad de géneros son: Asteraceae con 16 (9,47\%), Orchidaceae con 9 (5,32\%), Solanaceae con 9 (5,32\%), Fabaceae con $6(3,55 \%)$, Malvaceae con 5 (2,96\%), Bromeliaceae y Acanthaceae con 4 (2,37\%), Pteridaceae y Scrophulariaceae con 3 $(1,78 \%)$. Las familias con el mayor número de especies son: Asteraceae con 20 (7,75\%), Orchidaceae con 17 (6,59\%), Solanaceae con 14 (5,43\%), Pteridaceae con 11 (4,26\%), Fabaceae con 8 (3,10\%), Malvaceae, Scrophulariaceae y Bromeliaceae con 6(2,33\%) y Acanthaceae con 4 (1,55\%). El resto presenta menos de tres géneros y seis especies por 


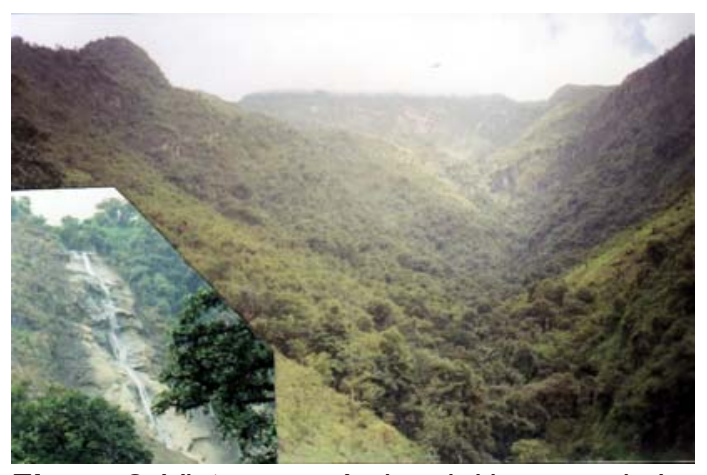

Figura 2. Vista panorámica del bosque de La Oscurana, parte inferior izquierda detalle de la catarata «El Chivo».

familia. Esta relación proporcional concuerda con otros inventarios realizados en las vertientes occidentales (Sagástegui \& Dillon, 1991; Cano \& Valencia, 1992; Dillon, 1993; Sagástegui, 1995; Sagástegui et al., 1995; Dillon et al., 2002).

Se observa una ampliación de la distribución geográfica de ciertas especies, antes sólo conocidas y endémicas al bosque de Monteseco (Weigend \& Rodríguez, 1998; Hensold, 1999; Sagástegui et al., 2003), tales como Nasa insignis Weigend \& E. Rodr. (Fig. 3) y Nasa humboltiana (Urb. \& Gilg) Weigend subsp. obliqua Dostert \& Weigend (Loasaceae), Trixis montesecoensis M.O. Dillon \& Sagást. (Asteraceae), Hydrocotyle sagasteguii Const. \& M.O. Dillon (Apiaceae), Miconia laciniata Wurdack (Melastomataceae), Tetrapterys dillonii W.R. Anderson (Malpighiaceae) y otras recientemente descritas como Alsophila mostellaria M. Lehnert (Cyatheaceae) de distribución más amplia. Esta diversidad excepcional, más sureña, sustenta aún mejor que la zona Amotape-Huancabamba sería una zona biogeográfica propia (Weigend, 2002).

La fisonomía del bosque montano La Oscurana está reflejada por los variados hábitos de crecimiento (Tabla 1). La mayoría de las 85 familias están ubicadas en seis categorías. Las hierbas incluyen 29 (34\%) familias, arbustos con 14 (16\%), árboles con 9 (11\%), trepadoras/lianas con $9(11 \%)$, hierbas/arbustos con 7 (8\%) y hierbas/epífitas con $5(6 \%)$ familias. Un menor porcentaje está representado por hierbas/trepadores (4 familias), hemiepífitos (2 familias), árboles/trepadores (2 familias), una familia con árboles/arbustos (Melastomataceae) y otra semiparásita (Loranthaceae). Las Asteraceae y Fabaceae presentan el mayor número de formas de vida; la primera es la más diversa del bosque e incluye árboles/arbustos/trepadoras o lianas y la segunda está conformada por hierbas/árboles/trepadoras.

Las hierbas y arbustos son las predominantes; esta aseveración siempre ha sido considerada en otros tipos de bosques tropicales (Gentry, 1988). Sin embargo, los árboles son la forma de vida más notoria del bosque y en los cuales se han centrado la mayoría de estudios en bosques tropicales (e.g. Gentry, 1992; León et al., 1992). Existen aún en este bosque relicto especies arbóreas pioneras típicas [e.g. Nectandra laurel Nees, Persea sp. 1, 2 (Lauraceae), Cedrela montana Moritz. Ex Turcz. y Guarea kunthiana A. Juss. (Meliaceae)], así como trepadoras/lianas [e.g. Neomortonia nummularia (Hanst.) Wiehler (Gesneriaceae), Passiflora spp. (Passifloraceae)]. Las epífitas [e.g. Orchidaceae, Bromeliaceae, varias Pteridophyta, musgos] presentan un bajo porcentaje, probablemente por la carencia de colecciones, en comparación con estudios en bosques similares, los cuales indican un elevado número juntamente con hierbas y arbustos (Gentry, 1988).

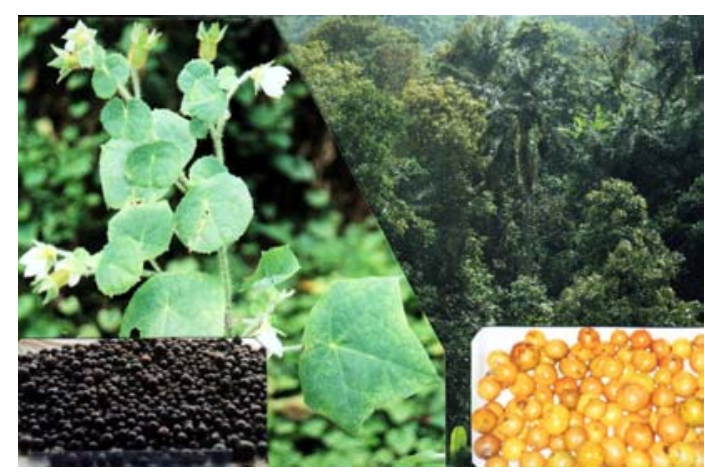

Figura 3. Detalle de la estructura del bosque. Izquierda: Especie endémica Nasa insignis (Loasaceae). Inferior izquierda: frutos (Myrtaceae). Inferior derecha: Frutos (Sapotaceae). 
Tabla 1. Composición florística y formas de vida existentes en el bosque de La Oscurana, distrito Bolívar, provincia de San Miguel de Pallaques, Dpto. de Cajamarca, 2002-2004.

\begin{tabular}{|c|c|c|c|c|c|}
\hline División/Familia & $\begin{array}{l}\text { Géneros } \\
\text { /Especies }\end{array}$ & $\begin{array}{l}\text { Formas } \\
\text { de vida** }\end{array}$ & División/Familia & $\begin{array}{l}\text { Géneros } \\
\text { /Especies }\end{array}$ & $\begin{array}{l}\text { Formas } \\
\text { de vida** }\end{array}$ \\
\hline PTERIDOPHYTA & & & LAURACEAE & $3 / 4$ & a \\
\hline ASPLENIACEAE & $1 / 3$ & h & LOASACEAE & $2 / 4$ & b,h \\
\hline BLECHNACEAE & $1 / 2$ & h & LOGANIACEAE & $1 / 1$ & b \\
\hline CYATHEACEAE & $1 / 1$ & a & LORANTHACEAE & $1 / 1$ & $\mathrm{p}$ \\
\hline DAVALLIACEAE & $1 / 2$ & $\mathrm{~h}$ & LYTHRACEAE & $1 / 2$ & $\mathbf{h}$ \\
\hline DENNSTAEDTIACEAE & $2 / 3$ & h & MALPIGHIACEAE & $2 / 3$ & $t$ \\
\hline DRYOPTERIDACEAE & $3 / 5$ & h, e & MALVACEAE & $5 / 6$ & b,h \\
\hline EQUISETACEAE & $1 / 2$ & h & MELASTOMATACEAE & $3 / 4$ & $a, b$ \\
\hline GLEICHENIACEAE & $1 / 2$ & h & MELIACEAE & $2 / 2$ & a \\
\hline HYMENOPHYLLACEAE & $1 / 1$ & h & MYRSINACEAE & $1 / 1$ & $\mathbf{a}$ \\
\hline LOPHOSORIACEAE & $1 / 2$ & h & MYRTACEAE & $3 / 3$ & a \\
\hline LYCOPODIACEAE & $1 / 1$ & h & NYCTAGINACEAE & $1 / 1$ & $\mathbf{b}$ \\
\hline POLYPODIACEAE & $2 / 5$ & h & ONAGRACEAE & $3 / 5$ & $\mathbf{b}$ \\
\hline PTERIDACEAE & $3 / 11$ & h & OXALIDACEAE & $1 / 3$ & h \\
\hline SELAGINELLACEAE & $1 / 1$ & h & PAPAVERACEAE & $1 / 1$ & b \\
\hline THELYPTERIDACEAE & $1 / 1$ & h & PIPERACEAE & $2 / 8$ & $h, b$ \\
\hline MAGNOLIOPHYTA & & & PLUMBAGINACEAE & $1 / 3$ & b \\
\hline & & & POLYGALACEAE & $1 / 1$ & $\mathbf{b}$ \\
\hline Magnoliopsida & & & POLYGONACEAE & $1 / 2$ & $\mathbf{h}$ \\
\hline ACANTHACEAE & $4 / 4$ & b,h & ROSACEAE & $2 / 2$ & $h, t$ \\
\hline ACTINIDIACEAE & $1 / 1$ & b & RUBIACEAE & $1 / 1$ & t \\
\hline AMARANTHACEAE & $2 / 4$ & $b, h$ & SAPINDACEAE & $2 / 2$ & $t, a$ \\
\hline ANACARDIACEAE & $1 / 1$ & a & SAPOTACEAE & $1 / 1$ & a \\
\hline APIACEAE & $2 / 2$ & h & SCROPHULARIACEAE & $3 / 6$ & h \\
\hline ARALIACEAE & $1 / 1$ & a & SOLANACEAE & $9 / 14$ & b,h \\
\hline ASCLEPIADACEAE & $1 / 1$ & t & STERCULIACEAE & $1 / 1$ & b \\
\hline ASTERACEAE & $16 / 20$ & $a, b, t$ & TROPAEOLACEAE & $1 / 1$ & $\mathbf{h}$ \\
\hline BEGONIACEAE & $1 / 3$ & h & URTICACEAE & $1 / 3$ & b \\
\hline BIGNONIACEAE & $2 / 2$ & $a, t$ & VERBENACEAE & $1 / 1$ & b \\
\hline BORAGINACEAE & $3 / 3$ & b & VITACEAE & $1 / 1$ & $\mathbf{t}$ \\
\hline BRASSICACEAE & $1 / 1$ & $\mathbf{h}$ & Liliopsida & & \\
\hline CACTACEAE & $1 / 1$ & h,e & ALSTROEMERIACEAE & $1 / 5$ & $h, t$ \\
\hline CAMPANULACEAE & $2 / 3$ & $h, t$ & AMARYLLIDACEAE & $3 / 3$ & h \\
\hline CARICACEAE & $1 / 2$ & b & ARACEAE & $1 / 5$ & h,e \\
\hline CARYOPHYLLACEAE & $1 / 2$ & $\mathbf{h}$ & ARECACEAE & $1 / 1$ & a \\
\hline CLUSIACEAE & $1 / 1$ & he & BROMELIACEAE & $4 / 6$ & h,e \\
\hline CONVOLVULACEAE & $2 / 2$ & $\mathbf{t}$ & COMMELINACEAE & $2 / 3$ & h \\
\hline CRASSULACEAE & $1 / 1$ & $\mathbf{h}$ & CYPERACEAE & $1 / 1$ & $\mathbf{h}$ \\
\hline CUCURBITACEAE & $3 / 3$ & $\mathbf{t}$ & DIOSCOREACEAE & $1 / 2$ & h \\
\hline ERICACEAE & $1 / 1$ & he & IRIDACEAE & $1 / 1$ & h \\
\hline EUPHORBIACEAE & $2 / 2$ & b & LILIACEAE & $1 / 1$ & h \\
\hline FABACEAE & $6 / 8$ & $a, h, t$ & ORCHIDACEAE & $9 / 17$ & $h, e$ \\
\hline GESNERIACEAE & $1 / 1$ & t & POACEAE & $3 / 3$ & $t, h$ \\
\hline LAMIACEAE & $2 / 4$ & b,h & SMILACACEAE & $1 / 1$ & $t$ \\
\hline
\end{tabular}

** Leyenda: $\mathrm{a}=$ árbol, palmera, helecho arbóreo; $\mathrm{b}=$ arbusto; e=epífito; $\mathrm{h}=$ hierba; he=hemiepífito o apoyante; $\mathrm{p}=$ semiparásita; $\mathrm{t}=$ trepadora o liana.

En este contexto, en un futuro cercano se puede aprovechar el magnífico entorno paisajístico-turístico que presenta, así como también realizar investigación científica (creación de una estación biológica). El uso directo de biodiversidad en La Oscurana puede lograrse mediante el manejo sustentable de re- cursos: alimenticios [e. g. frutos de Carica candicans A. Gray «checa» y C. stipulata Badillo «timbura» (Caricaceae), Myrcianthes sp. «arrayán», Eugenia sp. «lanche»y Myrcia fallax (Richd.) DC. «llama» (Myrtaceae), Chrysophyllum sp. (Sapotaceae), Allophyllus sp. «mote-mote» 
(Fig. 3)], medicinales [e. g. Dioscorea sp. 1, sp. 2 «papa madre» (Dioscoreaceae), Alternanthera porrigens (Jacquin) Kuntze «moradilla» (Amaranthaceae), Begonia acerifolia Kunth «contra hechizo» (Begoniaceae), Piper spp. «matico», Oncidium spp. «vaquita» (Orchidaceae)], industriales [e.g. Boconia integrifolia H.B.K. «español» (Papaveraceae, usado como tinte], madereros [e.g. Cordia alliodora (R. \& P.) Oken «laurel»(Boraginaceae), Nectandra laurel Nees «roble cute», Persea sp.1 «roble amarillo» (Lauraceae), Cedrela montana J. Moritz ex Turcz. «cedro negro» (Meliaceae), una Arecaceae denominada «shonta», etc., utilizados en la construcción de viviendas, muebles, utensilios caseros y leña] y ornamentales [e.g. Anthurium spp. (Araceae), Bomarea torta (H.B.K.) Herb. y B. ovata (Cav.) Mirbel (Alstroemeriaceae)].

\section{Estrategias y conservación del bosque}

Una estrategia coherente de conservación integral del bosque debe involucrar:

1) Científicos, (de las universidades de la región, ONG u otros) los cuales realizarían estudios sobre la biología y ecología de este frágil ecosistema, además de los estudios necesarios para la reforestación con especies nativas una vez identificadas y reconocidas mediante estudios florísticos. A esto se sumaria la creación de un banco de semillas.

Tabla 2. Riqueza genérica y específica de las familias más diversas del bosque de La Oscurana, distrito Bolívar, provincia de San Miguel de Pallaques, Departamento de Cajamarca.

\begin{tabular}{lrrrcc}
\hline Familia & \multicolumn{2}{c}{ Géneros } & & \multicolumn{2}{c}{ Especies } \\
\cline { 2 - 3 } \cline { 6 - 7 } & Número & \% & & Número & \% \\
\hline Asteraceae & 16 & 9,47 & & 20 & 7,75 \\
Orchidaceae & 9 & 5,32 & & 17 & 6,59 \\
Solanaceae & 9 & 5,32 & & 14 & 5,43 \\
Fabaceae & 6 & 3,55 & & 8 & 3,10 \\
Malvaceae & 5 & 2,96 & & 6 & 2,33 \\
Bromeliaceae & 4 & 2,37 & & 6 & 2,33 \\
Acanthaceae & 4 & 2,37 & & 4 & 1,55 \\
Pteridaceae & 3 & 1,78 & & 11 & 4,26 \\
Scrophulariaceae 3 & 1,78 & & 6 & 2,33 \\
\hline
\end{tabular}

2) El Estado, a través del Ministerio de Agricultura y el Instituto Nacional de Recursos Naturales (INRENA) con la implementación de adecuadas leyes de protección y estrategias para su cumplimiento.

3) El gobierno regional de Cajamarca y el gobierno distrital, asesorando, proponiendo proyectos, creando conciencia conservacionista (educación ambiental) y trabajando conjuntamente con la población del distrito de Bolívar.

El distrito de Bolívar será beneficiado directo por los beneficios que ofrece el bosque. Los pobladores después de solucionar sus necesidades económicas prioritarias utilizando los recursos en forma sustentable, podrán interiorizar la necesidad de aprovechar los recursos del bosque, pero a la vez conservarlo. De esta forma se garantizaría la protección de este ecosistema de una protección integral y sustentable. Finalmente, se plantea la urgente delimitación del bosque La Oscurana, la creación de la zona de amortiguamiento y declararla zona de protección o intangible.

\section{Agradecimientos}

Agradecemos a los directores y curadores de los herbarios HAO, HUT, PRG y USM. También nuestra gratitud para A. Sagástegui (HAO), A. Hofreiter (MSB), S. Llatas (PRG) y M. Chanco (USM) por el apoyo en las determinaciones taxonómicas, M. Weigend (Berlín) por la revisión y sugerencias al manuscrito, A. Hofreiter (Múnich) por la elaboración del abstract, A. Sánchez (Bolívar) por su ayuda permanente durante el estudio de campo y E. Barboza (Bolívar) por brindar información etnobotánica.

\section{Literatura citada}

Alvítez, E. 1977. Aspectos Fitogeográficos de la Provincia de San Miguel, Dpto. Cajamarca. Tesis para optar el grado de Bachiller en Ciencias Biológicas, Universidad Nacional de Trujillo, Perú. 50 p.

Brako, L. \& J. Zarucchi (eds.). 1993. Catálogo de las Angiospermas y Gimnospermas del Perú. Monogr. Syst. Bot. Missouri Bot. Garden. Vol 45.

Cano, A. \& N. Valencia. 1992. «Composición florística de los bosques nublados secos de la vertiente occidental de los Andes peruanos». En Young, K.R. \& N. Valencia (eds.) Biogeografía, 
Ecología y Conservación del Bosque Montano en el Perú. Memorias del Museo de Historia Natural UNMSM, Lima. 21: 171-180

Cronquist, A. 1981. An Integrated System of Classification of Flowering Plants. Nueva York: Columbia University Press.

Cronquist, A. 1988. The Evolution and Classification of Flowering Plants. 2. ${ }^{\mathrm{a}}$ edición. Nueva York: The New York Botanical Garden.

Dillon, M.O. 1993. «Análisis florístico del Bosque Monteseco (Cajamarca, Perú) e implicancias para su comparación». Arnaldoa, vol II (2): 29-42.

Dillon, M.O. 1994. «Bosques húmedos del norte del Perú». Arnaldoa, vol 2(1): 29-41.

Dillon, M.O., A. Sagástegui, I. Sánchez, S. Llatas \& N. Hensold. 1995. Floristic Inventory and Biogeographic Analysis of Montane Forest Northwestern Peru. Nueva York: Edit. S. Churchill et al., New York Botanical Garden, pp. 251-269.

Dillon, M.O., I. Sánchez, G. Iberico, A. Sagástegui, M. ZAPATA \& M. KAWASAKI. 2002. «Biogeografía, diversidad florística y endemismos en dos tipos de bosques en el norte del Perú».. Arnaldoa 9(2): 111-120.

Dourejanni, M. 1990. Amazonía ¿Qué hacer? Iquitos: Centro Estudios Teológicos de la Amazonía (CETA), 444 p.

Foster, B. R., N. C. Hernández, E., E. K.Kakudidi y R. J. Burnham. 1995. Un método de transectos variables para la evaluación rápida de comunidades de plantas en los trópicos. Manuscrito no publicado. Chicago: Environmental and Conservation Programs, Field Museum of Natural History; and Washington, D. C.: Conservation Biology, Conservation International.

Gentry, A. H. 1988. «Patters of diversity and floristic composition on environmental and geographical gradients». Ann. Missouri Bot. Garden 75: 1-34.

Gentry, A. H. 1992. «Diversity and floristic composition of Andean forests of Peru and adjacent countries: implications for their conservation». En Young, K.R. \& N. Valencia (Eds.) Biogeografia, Ecología y Conservación del Bosque Montano en el Perú. Memorias del Museo de Historia Natural UNMSM, Lima. 21: 11-29.

Hensold, N. 1999. Las Angiospermas endémicas del Dpto. de Cajamarca, Perú. Arnaldoa 6(2): 141-184.

Holmgren, P.; N. H. Holmgren \& L. C. Barnett. 1990. Index Herbariorum. 1. ${ }^{\text {a }}$ parte, The Herbaria of the world, $8{ }^{a}$ edición. Nueva York: New York Botanical Garden

León, B.; K. Young \& L. Brako. 1992. «Análisis de la composición florística del bosque montano oriental del Perú». En Young, K.R. \& N. Valencia (Eds.) Biogeografía, Ecología y Conser- vación del Bosque Montano en el Perú. Memorias del Museo de Historia Natural UNMSM, Lima. 21: 141-164.

López, A. 1993. «Catálogo de la Flora del Departamento de La Libertad (primera parte)». Arnaldoa 1(3): 15-44.

López, A. 1995a. «Catálogo de la Flora del Departamento de La Libertad (segunda parte)». Arnaldoa 3(1): 59-91.

López, A. 1995b. Catálogo de la Flora del Departamento de La Libertad (tercera parte). Arnaldoa 3(2): $35-72$.

López, A. 1998a. «Catálogo de la Flora del Departamento de La Libertad (cuarta parte)». Arnaldoa 5(1): 93-125.

López, A.1998b. «Catálogo de la Flora del Departamento de La Libertad (quinta parte)». Arnaldoa 5(2): 215-263.

Luteyn, J.L. 1992. «A new Ceratostema (Ericaceae: Vaccinieae) from northern Peru». Bull. Torrey Bot. Club 119(3): 314-315.

Macbride, F. 1936 y sgtes. Flora of Peru. Pub. Field Museum Nat. Hist. Vol.13.

Ritter, F. 1981. «Kakteen in Südamerika». Peru. Band 4, pp. 1239-1692.

Robinson, H. 1993. «A review of the genus Critoniopsis in Central and South America (Vernonieae: Asteraceae)». Proc. Biol. Soc. Wash. 106(3):620.

Rodríguez, E., S. Leiva \& E. Alvítez. 2000. «Avances en el estudio de la Flora Vascular de la Prov. de San Miguel, Dpto. Cajamarca-Perú». En Libro de Resúmenes del VIII CONABOT, Arequipa, p. 41.

Rodríguez, E., E. Alvítez \& S. Leiva. 2002. «Estudio de la Flora Vascular de la Provincia de San Miguel Pallaques, Dpto. Cajamarca-Perú (II)». En Libro de Resúmenes del IX CONABOT, Iquitos, p. 85.

Rodríguez, E. \& R. Rojas. 2002. El Herbario, Administración y Manejo de Colecciones Botánicas. Edit. Missouri Bot. Garden. 56 p.

Sagástegui, A. \& I. Sánchez. 1991. «Una nueva especie de Chuquiraga (Asteraceae-Mutisieae)». Arnaldoa. 1(2): 1-4.

Sagástegui, A. \& M.O. Dillon. 1991. «Inventario Preliminar de la Flora del Bosque de Monteseco». Arnaldoa. 1(1): 35-52.

Sagástegui, A. 1994. «Flora endémica de los Andes Norperuanos». Arnaldoa. 2(1): 43-63.

Sagástegui, A. 1995. Diversidad Florística de Contumazá. Trujillo: Fondo Editorial Univ. Antenor Orrego.

Sagástegui, A., S. Leiva, P. Lezama, N. Hensold \& M.O. Dillon. 1995. «Inventario Preliminar de la Flora del Bosque de Cachil». Arnaldoa. 3 (2): 19-34.

Sagástegui, A., M.O. Dillon, I. Sánchez, S. Leiva \& P. Lezama. 1999. Diversidad Florística del Norte del Perú. Tomo I. Trujillo: Edit. Graficart. 
Sagástegui, A.; I. Sánchez; M. Zapata \& M.O. Dillon. 2003. Diversidad Florística del Norte del Perú. Bosques Montanos. Trujillo: tomo II. Edit. Graficart.

Vásquez, R. 1995. «Árboles de la Amazonía Nor Oriental del Perú: Diversidad, destrucción y conservación». Arnaldoa. 3(2): 73-86.

Tryon, R. M. \& A. F. Tryon. 1982. Ferns and Allied Plants with Special Reference to Tropical America. Nueva York: Edit. Springer-Verlag.

Tryon, R. \& R. Stolze. 1989-1994. «Pteridophyta of Peru». Fieldiana, Bot. n.s. Vols. 20, 22, 27, 29, 32, 34.
Weberbauer, A. 1945. «El mundo vegetal de los Andes Peruanos». Est. Experim. La Molina, Lima.

Weigend, M.; E. Rodríguez \& N. Dostert. 1998. «Nasa insignis yNasa glandulossima(Loasaceae: Loasoideae), dos nuevas especies con hojas peltadas del Norte de Perú». Arnaldoa 5(2): 151-157.

Weigend, M. 2002. «Observations on the Biogeography of the Amotape-Huancabamba Zone in Northern Peru». The Botanical Review 68(1): 38-54.

Womersley, J. 1981. Plant collecting and herbarium development. FAO Plant Prod. and Protec. Paper. N. ${ }^{\circ} 33$.

Anexo 1. Lista de taxa presentes en el bosque relicto de La Oscurana (Distrito Bolivar, Provincia San Miguel, CAJAMARCA) (Colectores : A. Juarez et al. ; Herbarios: HAO, HUT, PRG, USM, por rep.)

\section{PTERIDOPHYTA}

ASPLENIACEAE

Asplenium abscissum Willd.

Asplenium cuspidatum Lam.

Asplenium sp.

BLECHNACEAE

Blechnum occidentale L.

Blechnum cordatum (Desv.) Hieron.

CYATHEACEAE

Alsophila mostellaria M. Lehnert

DAVALLIACEAE

Nephrolepis pectinata (Willd.) Schott

Nephrolepis sp.

\section{DENNSTAEDTIACEAE}

Dennstaedtia arborescens (Willd.) Ekman ex Maxon

Dennstaedtia punctilobula (Michx.) T. Moore

Pteridium aquilinum (L.) Kuhn

\section{DRYOPTERIDACEAE}

Dryopteris sp.

Elaphoglossum glossophyllum Hieron.

Elaphoglossum latifolium (Sw.) J. Sm.

Tectaria lizarzaburui (Sodiro) C. Chr.

Tectaria $\mathrm{sp}$.

\section{EQUISETACEAE}

Equisetum bogotense Kunth

Equisetum giganteum L.

GLEICHENIACEAE

Gleichenia revoluta Kunth

Gleichenia sp.

\section{HYMENOPHYLLACEAE}

Hymenophyllum myriocarpum Hook.

\section{LOPHOSORIACEAE}

Lophosoria quadripinnata (J.F. Gmel.) C. Chr.

Lophosoria sp.

\section{LYCOPODIACEAE}

Lycopodium clavatum $\mathrm{L}$

\section{POLYPODIACEAE}

Niphidium crassifolium (L.) Lellinger

Polypodium lasiopus Klotzsch

Polypodium thyssanolepis A. Braun ex Klotzsch

Polypodium sp. 1

Polypodium sp.2

\section{PTERIDACEAE}

Adiantum concinnum Humb. \& Bonpl. ex Willd.

Adiantum digitatum Hook.

Adiantum patens Willd.

Adiantum poiretii Wikstr.
Adiantum orbignyanum Kuhn

Adiantum raddianum C. Presl

Adiantum sp1.

Adiantum sp2.

Pityrogramma tartarea (Cav.) Maxon

Pityrogramma trifoliata (L.) R.M. Tryon

Pteris muricata Hook.

SELAGINELLACEAE

Selaginella silvestris Aspl.

THELYPTERIDACEAE

Thelypteris patens (Sw.) Small

\section{MAGNOLIOPHYTA}

\section{Magnoliopsida}

\section{ACANTHACEAE}

Aphelandra acanthifolia Hook.

Diclipthera sp.

Justicia sp.

Beloperone sp.

ACTINIDIACEAE

Saurauia sp.

\section{AMARANTHACEAE}

Alternanthera mexicana (Schltdl.) Hieron.

Alternanthera porrigens (Jacq.) Kuntze

Alternanthera sp.

Iresine diffusa Humb. \& Bonpl. ex Willd.

ANACARDIACEAE

Mauria heterophylla Kunth

APIACEAE

Arracacia elata $\mathrm{H}$. Wolff

Hydrocotyle sagasteguii Constance \& M.O. Dillon

ARALIACEAE

Oreopanax sp.

ASCLEPIADACEAE

Cynanchum $s p$.

ASTERACEAE

Acmella oppositifolia (Lam.) R.K. Jansen

Ageratina articulata (Sch. Bip. ex Hieron.) R. M. King \& H. Rob. Austroeupatorium sp.

Baccharis auriculigera Hieron.

Baccharis latifolia (Ruiz \& Pav.) Pers.

Baccharis sp.1

Baccharis sp.2

Barnadesia hutchisoniana Ferreyra

Bidens squarrosa Kunth 
Chromolaena odorata (L.) R.M. King \& H. Rob. Jungia discolor Muschl.

Mikania sp.

Monactis flaverioides Kunth

Pentacalia reflexa (Kunth) Cuatrec.

Senecio sp.

Trixis montesecoensis Sagást. \& M.O. Dillon

Verbesina sp.

Vernonanthura patens (Kunth) H. Rob.

Vernonia canescens Kunth

Vernonia scorpioides (Lam.) Pers.

\section{BEGONIACEAE}

Begonia acerifolia Kunth

Begonia sp.1

Begonia sp.2

\section{BIGNONIACEAE}

Delostoma integrifolium D. Don

Tecoma sambucifolia Kunth

BORAGINACEAE

Cordia alliodora (Ruiz \& Pav.) Oken

Heliotropium rufipilum (Benth.) I.M. Johnst.

Tournefortia longifolia Ruiz \& Pav.

\section{BRASSICACEAE}

Cremolobus peruvianus (Lam.) DC.

CACTACEAE

Rhipsalis micrantha (Kunth) DC.

\section{CAMPANULACEAE}

Centropogon pilosulus E. Wimm.

Centropogon vernicosus A. Zahlbruckner

Siphocampylus sp.

CARICACEAE

Carica stipulata V.M. Badillo

Carica candicans A. Gray

CARYOPHYLLACEAE

Drymaria cf. cordifolia (L.) Willd.

Drymaria villosa Cham. \& Schldl

CLUSIACEAE

Clusia sp.

\section{CONVOLVULACEAE}

Jacquemontia prominens Helwig

Ipomea $\mathrm{sp}$.

CRASSULACEAE

Bryophyllum pinnatum (Lam.) Oken

CUCURBITACEAE

Cyclanthera brachybotrys (Poepp. \& Endl.) Cogn.

Melothria pendula L.

Sicyos macrocarpus Cogn.

\section{ERICACEAE}

Ceratostema callistum A.C. Sm.

\section{EUPHORBIACEAE}

Croton abutiloides Kunth

Dalechampia aristolochiifolia Kunth

\section{FABACEAE}

Amicia glandulosa Kunth

Desmodium affine Schltdl.

Desmodium vargasianum B.G. Schub.

Desmodium sp.1

Erythrina edulis Triana ex Micheli

Inga oerstediana Benth.

Otholobium pubescens (Poir.) J.W. Grimes

Phaseolus polyanthus Greenm.

\section{GESNERIACEAE}

Neomortonia nummularia (Hanst.) Wiehler

LAMIACEAE

Salvia florida Benth.
Salvia sp.1

Salvia sp. 2

Satureja revoluta (Ruiz \& Pav.) Briq.

LAURACEAE

Beilschmiedia sulcata (Ruiz \& Pav.) Kosterm.

Nectandra laurel Klotzsch ex Nees

Persea sp. 1

Persea sp.2

LOASACEAE

Klaprothia fasciculata (C. Presl) Poston

Nasa humboldtiana (Urb. \& Gilg) Weigend subsp. obliqua Dostert \& Weigend

Nasa insignis Weigend \& E. Rodr.

Nasa olmosiana (Gilg ex J.F. Macbr.) Weigend

LOGANIACEAE

Buddleja americana $\mathrm{L}$

LORANTHACEAE

Tristerix longebracteatus (Desr.) Barlow \& Wiens LYTHRACEAE

Cuphea strigulosa Kunth

Cuphea sp.

MALPIGHIACEAE

Stigmaphyllon bogotense Triana \& Planch.

Stigmaphyllon sp.

Tetrapterys dillonii W.R. Anderson

MALVACEAE

Abutilon dianthum C. Pres1

Bastardia sp.

Fuertesimalva $\mathrm{sp}$.

Pavonia sepium A. St.-Hil.

Sida repens Dombey ex Cav.

Sida rhombifolia L.

\section{MELASTOMATACEAE}

Axinaea cf. nitida Cogn.

Axinaea sp1.

Miconia laciniata Wurdack

Tibouchina laxa (Desr.) Cogn.

MELIACEAE

Cedrela montana Moritz ex Turcz.

Guarea kunthiana A. Juss.

MYRSINACEAE

Geisanthus sp.

MYRTACEAE

Eugenia quebradensis McVaugh

Myrcia fallax (Rich.) DC.

Myrcianthes sp.

NYCTAGINACEAE

Colignonia parviflora (Kunth) Choisy

ONAGRACEAE

Fuchsia ayavacensis Kunth

Fuchsia sp.1

Ludwigia peruviana (L.) H. Hara

Ludwigia sp.1

Oenothera rosea L'Hér. ex Aiton

OXALIDACEAE

Oxalis spiralis Ruiz \& Pav. ex G. Don

Oxalis sp.1

Oxalis sp.2

\section{PAPAVERACEAE}

Bocconia integrifolia Humb. \& Bonpl.

PASSIFLORACEAE

Passiflora mollissima (Kunth) L.H. Bailey

Passiflora aff. montana (Barb. Rodr.) Harms

Passiflora sp. 1

Passiflora sp.2 
PHYTOLACCACEAE

Phytolacca weberbaueri H. Walter PIPERACEAE

Peperomia alata Ruiz \& Pav.

Peperomia fraseri C. DC.

Peperomia sp.1

Peperomia sp.2

Piper acutifolium Ruiz \& Pav.

Piper aequale Vahl

Piper sp. 1

Piper sp.2

PLUMBAGINACEAE

Plumbago scandens L.

Plumbago coerulea Kunth

Plumbago sp.1

POLYGALACEAE

Monnina paniculata Benth.

POLYGONACEAE

Polygonum hydropiperoides Michx.

Polygonum sp.

\section{ROSACEAE}

Duchesnea indica (Andrews) Focke

Rubus robustus C. Presl

\section{RUBIACEAE}

Psychotria sp.

SAPINDACEAE

Allophylus densiflorus Radlk.

Serjania sp.

SAPOTACEAE

Chrysophyllum sp.

SCROPHULARIACEAE

Bartsia mutica (Kunth) Benth.

Calceolaria calycina Benth.

Calceolaria dichotoma Lam.

Calceolaria nivalis Kunth

Calceolaria pinnata $\mathrm{L}$.

Castilleja arvensis Schltdl. \& Cham.

\section{SOLANACEAE}

Acnistus arborescens (L.) Schltdl.

Browallia americana L.

Cestrum auriculatum L'Hér.

Cestrum tomentosum L.f.

Iochroma cornifolium (Kunth) Miers

Lycianthes radiata (Sendtn.) Bitter

Lycopersicon cf. hirsutum Dunal

Nicotiana tabacum L.

Nicotiana thyrsiflora Goodsp.

Physalis peruviana L.

Solanum caripense Dunal

Solanum oblongifolium Dunal

Solanum abitaguense S. Knapp

Solanum asperolanatum Ruiz \& Pav.

STERCULIACEAE

Byttneria lopez-mirandae Cristóbal

TROPAEOLACEAE

Tropaeolum fintelmannii Wagener ex Schltdl.

URTICACEAE

Pilea microphylla (L.) Liebm.

Pilea pubescens Liebm.

Pilea sp.

VERBENACEAE

Lantana sp.

VITACEAE

Cissus obliqua Ruiz \& Pav.

\section{Liliopsida}

\section{ALSTROEMERIACEAE}

Bomarea distichifolia (Ruiz \& Pavón) Baker

Bomarea torta (H.B.K.) Herb.

Bomarea ovata (Cav.) Mirb.

Bomarea superba Herb.

Bomarea sp.

\section{AMARYLLIDACEAE}

Eucrosia eucrosioides (Herb.) Pax

Leptochiton helianthus (Ravenna) Gereau \& Meerow

Stenomesson sp.

ARACEAE

Anthurium breviscapum Kunth

Anthurium soukupii Croat

Anthurium sp.1

Anthurium sp. 2

Anthurium sp. 3

ARECACEAE

Género 1

\section{BROMELIACEAE}

Guzmania monostachia (L.) Rusby ex Mez

Guzmania variegata L. B. Sm.

Pitcairnia lopezii L. B. Sm.

Tillandsia complanata Benth.

Tillandsia sagasteguii L. B. Sm.

Vriesea cf. cylindrica L. B. Sm.

COMMELINACEAE

Commelina sp. 1

Commelina sp. 2

Tinantia sp.

CYPERACEAE

Cyperus $s p$.

DIOSCOREACEAE

Dioscorea polygonoides Humb. \& Bonpl. ex Willd.

Dioscorea sp.

IRIDACEAE

Crocosmia x crocosmiiflora (Lemoine) N.E. Br..

LILIACEAE

Género 1

ORCHIDACEAE

Cranichis longipetiolata C. Schweinf.

Cranichis sp.

Encyclia sp.

Epidendrum gracillimum Reichb. f. \& Warcz.

Epidendrum sp.1

Epidendrum sp.2

Govenia sp.

Odontoglossum longipes Reichb. f. \& Warcz.

Oncidium macranthum Lindl.

Oncidium sp.1

Oncidium sp. 2

Pleurothallis spiralis (Ruiz \& Pav.) Lindl

Pleurothallis sp.1

Pleurothallis sp. 2

Pleurothallis sp.3

Stelis sp. 1

Telipogon sp. 1

POACEAE

Chusquea scandens Kunth

Cortaderia aristata Pilg.

Polypogon elongatus Kunth

SMILACACEAE

Smilax sp. 